\title{
JUVENILE CHRONIC INFLAMMATORY DEMYELINATING POLYNEUROPATHY (CIDP) EPIDEMIOLOGY IN SARDINIA, INSULAR ITALY.
}

Authors: Stefano Sotgiu, MD, ${ }^{1}$ Ilaria Onida, MD, ${ }^{1}$ Giorgio Magli, MD, ${ }^{1}$ Paolo Castiglia, MD, ${ }^{2}$ Marta Conti, MD,${ }^{1}$ Angela Nuvoli, MD,${ }^{1}$ Alessandra Carta, MD, ${ }^{1}$ Silvia Festa, MD,${ }^{1}$ Veronica Dessì, MD, ${ }^{1}$ Pietro Emilio Doneddu, MD, ${ }^{3}$ Eduardo Nobile-Orazio, MD. ${ }^{3}$

Affiliations: 1) Child Neuropsychiatry Unit Department of Medical, Surgical and Experimental Sciences, University of Sassari, Italy; 2) Hygiene and Preventive Medicine Unit, Department of Medical, Surgical and Experimental Sciences, University of Sassari, Italy; 3) Humanitas Clinical and Research Institute, Milan, Italy.

Emails: (SS) stefanos@ uniss.it, (IO) ilaonida91@hotmail.it, (GM) magli.giorgio@gmail.com, (PC), castigli@uniss.it, (MC) marta.conti7@gmail.com, (AN) angela.nu@hotmail.com, (AC) carta.ale84@gmail.com, (SF) silviafesta71@gmail.com, (VD) veronicadess @libero.it, (PED) pietro.doneddu@ humanitas.it, (EN-O) eduardo.nobile@unimi.it.

Location: The work has been mostly performed at the Child Neuropsychiatry Unit, University of Sassari, Italy

Running title: juvenile CIDP in Sardinia

Word count: 1701

number of references: 15

Number of figures/tables: 1 table, 1 figure

Corresponding author: Prof. Stefano Sotgiu, Unità Operativa Complessa di Neuropsichiatria Infantile, Dipartimento di Scienze Mediche, Chirurgiche e Sperimentali, Università di Sassari, Viale San Pietro, 42/B; I-07100 Sassari, Italy. Tel +39 079229322

Email: stefanos@uniss.it; stefano.sotgiu@aousassari.it 


\begin{abstract}
Background. Chronic inflammatory demyelinating polyradiculoneuropathy (CIDP) is a rare and disabling immunomediated radiculoneuropathy. Its worldwide epidemiology is heterogeneous and, in adults, CIDP prevalence varies from 0.6 to 9 cases per 100,000 population. Juvenile CIDP (jCIDP) is even rarer, with age-specific prevalence rates varying from 0.23 to 1.26 owing to different diagnostic criteria (AAN and EFNS/PNS), different age-grouping or, genuine differences.
\end{abstract}

Objectives. We assessed jCIDP incidence and prevalence in Sardinia, an area at very-high risk for autoimmune diseases, using comparable methods.

Design. The study area was the northern Sardinia, insular Italy, with 491,571 inhabitants and a pediatric population (0-18 years) of 79,086 individuals.

Results. On prevalence day (December 31, 2019) the total crude, age-specific prevalence rate were 6.32/100,000 according with AAN criteria, 7.58/100,000 population with ENMC criteria and 8.85/100,000 population with both 2006 and 2010 EFNS/PNS criteria. Crude mean incidence rate were 0.42/100,000/year with AAN criteria, 0.50/100,000/year with ENMC criteria and 0.59/100,000/year using 2006 and 2010 EFNS/PNS criteria. Of the eight patients, six had typical CIDP, one had MADSAM, and one CISP. Patient's disability was generally mild. Clinical course was progressive, monophasic or relapsing.

Conclusion. jCIDP prevalence and incidence rates in Sardinia were criteria-dependent, the lowest obtained when using AAN criteria, the highest using the EFNS/PNS. Nonetheless, even with the exclusion of the "possible" category, by using comparable methodology, prevalence rates in Sardinia are considerably higher than the range reported in all previous jCIDP studies.

Key words: CIDP, epidemiology, prevalence, incidence, Sardinia, diagnostic criteria. 


\section{INTRODUCTION}

Chronic inflammatory demyelinating polyradiculoneuropathy (CIDP) is an uncommon acquired immuno-mediated neuropathy, observed at all ages, causing limb weakness and/or sensory deficits. ${ }^{1}$ CIDP etiology remains elusive and preceding infections or vaccinations are only weak risk factors. ${ }^{1}$ Juvenile CIDP (jCIDP; age <19 years) seems to present different clinical features from the adult form, such as no gender predilection, rapid progression, motor predominance, greater likelihood of relapse, and better prognosis. ${ }^{2}$ Different diagnostic criteria for jCIDP have been proposed by the European Neuromuscular Center at its international (ENMC) workshop in $2000,{ }^{3}$ but so far they have not been used for epidemiological purposes. Their sensitivity is lower than that of the EFNS/PNS and AAN criteria, while specificity is higher. ${ }^{4}$ Other electrodiagnostic criteria have also been suggested as more specific in differentiating jCIDP from hereditary neuropathies, but they have not been formally approved by an international expert commission. ${ }^{4}$ Despite all these efforts, diagnosis of jCIDP is still challenging and investigations are rare. The variant forms described in adults have been rarely documented in jCIDP, although this may reflect the smaller number of cases. ${ }^{3}$ One Japanese study determined an age-specific (0-15 years) crude incidence rate of $0.06 / 100.000$ person/year. ${ }^{5}$ As shown on Fig. 1A, jCIDP prevalence rates are much lower than adults and, according to different age-groups and diagnostic criteria, they can vary from 0,00 (0-9 years) up to 1.26 (0-19 years) ${ }^{5-9}$ Aiming at a comparative analysis, we assessed jCIDP epidemiology in Sardinia, an area at very-high risk for pediatric neurological and systemic autoimmune diseases, ${ }^{10}$ using different existing diagnostic criteria, including the ENMC criteria.

\section{MATERIALS AND METHODS}

The study involved the province of Sassari, northern Sardinia, insular Italy, an area of around 7,600 $\mathrm{Km}^{2}$ which now includes the former province of Olbia-Tempio. The prevalence day was December 31, 
2019, when inhabitants were 491,571 with a pediatric population (0-19 years, $16 \%$ ) of 79,086 individuals (36,524 in the age-group 0-9 years and 42,562 between 10-19 years).

jCIDP patients were searched through a network of health-care workers located in the study area or immediate and adjacent areas, and referred to the Child Neuropsychiatry Unit, University Hospital of Sassari. Contributing centres were Pediatric and Neurological Divisions in Sassari, Ozieri and Olbia Hospitals. Provincial child neuropsychiatry outpatients services and rehabilitation centres were also contacted. Data were confirmed checking the regional centralized archive by using the national code RF0180.

All medical files, with results of electrophysiological studies, were included if diagnosis of CIDP, chronic inflammatory neuropathies, Guillain Barré syndrome, polyradiculoneuritis, polyneuritis or peripheral demyelinating disorder were reported within the age range 0-19 years. Patients whose differential diagnosis was missing, who did not satisfy published criteria for CIDP or bearing diagnoses of hereditary neuropathy, Charcot-Marie-Tooth, monoclonal gammopathy, paraneoplastic, metabolic, amyloid and diabetic neuropathy were ruled out from the subsequent analysis.

Collection of demography, date, location and symptoms at CIDP onset, date of diagnosis, course, treatments and the 0-12 scale score of the ONLS (Overall Neuropathy Limitation Scale) were included. ${ }^{11}$ AAN, 2006 and 2010 EFNS/PNS, and the diagnostic criteria for jCIDP proposed by the ENMC workshop were used to define jCIDP. ${ }^{3,12-14}$ Only 'probable' or 'definite' jCIDP cases according to the EFNS/PNS and AAN criteria or 'confirmed' cases according to the ENMC criteria were finally computed.

\section{STATISTICAL ANALYSIS}

Crude, age-specific, rates were calculated for the annual number of new cases (incidence) or the total jCIDP cases (prevalence) diagnosed and living in the period between 1 January 2005 and 31 December 2019 (15 years) in the study area; $95 \%$ confidence intervals $(95 \% \mathrm{CI})$ were computed. 


\section{RESULTS}

Ten patients were initially identified with a diagnosis of possible or suspected CIDP according to the treating neurologist and the aforementioned methods. Seven of these patients fulfilled the EFNS/PNS criteria, six patients fulfilled the ENMC criteria, and five patients the AAN criteria. All these three sets of diagnostic criteria require that the diagnosis of a pure sensory CIDP can be made when demyelinating features are observed not only on sensory but also on motor nerve conduction studies. ${ }^{3,12-14}$ When distal motor and sensory nerve conduction are normal, diagnosis of chronic immune sensory polyradiculopathy (CISP) can be made in a patient with sensory symptoms in a polyneuropathic distribution without weakness when (at least) two of the followings are present: abnormal somatosensory evoked potential (SSEP) not attributable to central involvement, MRI showing enlarged or gadolinium-enhanced nerve roots or plexuses, and CSF hyperproteinorrachia with normal cellular count. ${ }^{1}$ This is the case n. 8 (Table 1). One patient was excluded from the analysis because he only satisfied criteria for possible CIDP and another because she was living outside the study area on prevalence day.

All eight selected patients were of Sardinian descent, and were living in the study area also at the time of disease outset. Five out of 8 were males; mean age at onset was 12 years (range 4-17); disease duration ranged from 1 to 15 years (mean 7.6 years). Four out of eight have been evaluated in mainland Italian centres for a second, confirmatory, opinion.

On prevalence day (December 31, 2019) the total crude, age-specific prevalence rate was 6.32/100,000 (95\% CI 2.31-14.01/100,000) with AAN criteria, 7.58/100,000 population using the ENMC criteria (95\% CI 3.34-16.1/100.000), and 8.85/100,000 population using both 2006 and 2010 EFNS/PNS criteria (95\% CI 4.56-17.32/100.000); prevalence estimates for age-subgroups 0-9 years and 10-19 years are reported on Fig. 1B.

As for clinical details (Table 1): Six patients had typical CIDP, one had a multifocal acquired demyelinating sensory and motor neuropathy (MADSAM), and one CISP. Two cases had their onset at age 4 and 5 years, respectively. After a mean 7.6-years follow-up, patient's disability ${ }^{11}$ was mild (score $\leq 5$ ) 
in $6(75 \%)$ and moderate (score 6) in $2(25 \%)$. CIDP course, ${ }^{1}$ was relapsing in 3 (at least two clinical deteriorations longer than 7 days after a remission lasting more than four weeks), monophasic in 2 cases (stable for at least 6 months, with no relapses) and progressive in the remaining 3 cases. Crude mean annual incidence rate was 0.59/100,000/year (95\% CI 0.32-2.03/100,000) using 2006 and 2010 EFNS/PNS criteria, 0.50/100,000/year using the ENMC criteria (95\% CI 0.29-1.70/100,000 and 0.42/100,000/year with AAN criteria (95\% CI 0.18-1.40/100,000).

\section{DISCUSSION}

Prevalence estimation of jCIDP differs worldwide owing to different case definition, different ascertainment, different age-grouping or because of genuine differences (Figure 1) ${ }^{5-9}$ In the adult form of CIDP, the EFNS/PNS criteria are preferred to the AAN criteria because are more sensitive but still specific. ${ }^{1,2,8}$ The AAN electrophysiological criteria require the presence of at least three demyelinating features in two or more motor nerves (except for conduction block which is sufficient in only one nerve), whereas the EFNS/PNS criteria require the presence of only one demyelinating abnormality in two nerves for a definite diagnosis. One epidemiologic study on adult and jCIDP showed that using the two diagnostic criteria in the same population, marked differences in prevalence and incidence figures are found. ${ }^{8}$ Another study compared the two sets of criteria in jCIDP finding similar sensitivity and specificity values. ${ }^{4}$ The ENMC criteria for jCIDP are more specific than EFNS/PNS or AAN but less sensitive. ${ }^{4}$ These criteria were specifically designed for jCIDP, a category of patients where the question about the etiology of the demyelinating neuropathy is more difficult to answer. Our jCIDP prevalence rates were criteria-dependent, the lowest obtained when using AAN criteria, the highest using the EFNS/PNS, and those obtained with the ENMC in the middle. Nonetheless, compared to former studies, even with the exclusion of the "possible" category, jCIDP frequency in Sardinia is the highest so far described using both the AAN and EFNS/PNS criteria. Using comparable methodology, prevalence rates in Sardinia are considerably higher than the range reported in previous jCIDP studies. ${ }^{5-9}$ 
This is consistent with immunogenetic and epidemiological studies indicating Sardinians to bear a worldwide primacy for autoimmune/immune-mediated diseases like multiple sclerosis, systemic lupus erythematous, type 1 diabetes, ${ }^{10}$ perhaps as a trade-off of the evolutionary response against malaria. ${ }^{10}$ It would be helpful to perform a CIDP epidemiological study also in adult population to make proper comparative analyses with other populations.

The number of patients is too limited to make a detailed phenotypical comparison. CIDP has atypical presentations in as much as $39 \%$ of cases, including pure sensory or motor forms. ${ }^{1}$ Although the frequency of atypical variants in jCIDP seems lower than that found in the adult forms, ${ }^{3}$ two cases (25\%) have been found in our study: one MADSAM, the most frequent CIDP variant, and one CISP, the least common. ${ }^{1}$ Recently, the presence of antibodies against proteins of the paranode and node of Ranvier have been also demostrated in some patients with jCIDP. ${ }^{15}$ Clinical course in our patients was either progressive, monophasic or relapsing. Our cases displayed an overall mild disability by ONLS, although two of them had been unable to walk independently at some point in their disease. This is in line with previous studies showing a worse functional scores at peak severity and better long-term prognosis in jCIDP compared to adult CIDP. ${ }^{2}$

In conclusion, our study confirms the increased jCIDP prevalence with age, the low disability of the juvenile form with only half patients on maintenance treatment (4/8), and the high susceptibility of the Sardinian population to autoimmune neurological diseases. Our work suffers from limitations because, in retrospective studies, data collection is not always complete; also, neurological examinations were not uniformly performed and relied on the skill of the treating neurologist; moreover, information was collected from multiple sources and reports of nerve conduction studies were not standardised. In addition, given the relatively small area of assessment, the results of this study need to be confirmed in a larger catchment area. This could be useful to calculate disease-related costs in Sardinia and to plan adequate medical services. 


\section{ACKNOWLEDGMENTS}

The authors are grateful to Dr. Giovanni Masala, laboratory of Clinical Neurophysiology, Unit of Neurology, University-Hospital, Sassari, Italy for helpful discussion and technical assistance.

\section{REFERENCES}

1. Doneddu PE, Cocito D, Manganelli F, et al. Atypical CIDP: diagnostic criteria, progression and treatment response. Data from the Italian CIDP Database. J Neurol Neurosurg Psychiatry 2018;90:125-132.

2. Hattori N, Misu K, Koike H, et al. Age of onset influences clinical features of chronic inflammatory demyelinating polyneuropathy. J Neurol Sci 2001;184:57-63.

3. Nevo Y, Topaloglu H. 88th ENMC international workshop: childhood chronic inflammatory demyelinating polyneuropathy (including revised diagnostic criteria), Naarden, The Netherlands, December 8-10, 2000. Neuromuscul Disord 2002;12:195-200.

4. Potulska-Chromik A, Ryniewicz B, Aragon-Gawinska K, et al. Are electrophysiological criteria useful in distinguishing childhood demyelinating neuropathies? J Peripher Nerv Syst 2016;21:22-26.

5. Iijima M, Koike H, Hattori N, et al. Prevalence and incidence rates of chronic inflammatory demyelinating polyneuropathy in the Japanese population. J Neurol Neurosurg Psychiatry 2008;79:10401043.

6. Mahdi-Rogers M, Hughes RA. Epidemiology of chronic inflammatory neuropathies in southeast England. Eur J Neurol 2014; 21:28-33.

7. McLeod JG, Pollard JD, Macaskill P, et al. Prevalence of chronic inflammatory demyelinating polyneuropathy in New South Wales, Australia. Ann Neurol 1999;46:910-913.

8. Rajabally YA, Simpson BS, Beri S, et al. Epidemiologic variability of chronic inflammatory demyelinating polyneuropathy with different diagnostic criteria: study of a UK population. Muscle Nerve 2009;39:432-438. 
9. Chio A, Cocito D, Bottacchi E, et al. Idiopathic chronic inflammatory demyelinating polyneuropathy: an epidemiological study in Italy. J Neurol Neurosurg Psychiatry 2007;78:1349-1353.

10. Dell'Avvento S, Sotgiu MA, Manca S, et al. Epidemiology of multiple sclerosis in the pediatric population of Sardinia, Italy. Eur J Pediatr 2016;175:19-29.

11. Graham RC, Hughes RA. A modified peripheral neuropathy scale: the Overall Neuropathy Limitations Scale. J Neurol Neurosurg Psychiatry 2006;77:973-976.

12. Ad Hoc Subcommittee of the American Academy of Neurology AIDS Taskforce. Research criteria for diagnosis of chronic inflammatory demyelinating polyneuropathy (CIDP). Neurology $1991 ; 41: 617-618$

13. Hughes RA, Bouche P, Cornblath DR, Evers E, Hadden RD, Hahn A, et al. European Federation of Neurological Societies/Peripheral Nerve Society guidelines on management of chronic inflammatory demyelinating polyneuropathy: report of a joint task force of the European Federation of Neurological Societies and the Peripheral Nerve Society. Eur J Neurol 2006;13:326 -332

14. Van den Bergh PY, Hadden RD, Bouche P, et al.; European Federation of Neurological Societies; Peripheral Nerve Society. European Federation of Neurological Societies/Peripheral Nerve Society guideline on management of chronic inflammatory demyelinating polyradiculoneuropathy: report of a joint task force of the European Federation of Neurological Societies and the Peripheral Nerve Society - first revision. Eur J Neurol. 2010 Mar;17(3):356-63.

15. Carrera-García L, Natera-de Benito D, Lleixà C, et al. Chronic inflammatory demyelinating polyneuropathy associated with contactin- 1 antibodies in a child. Neurol Neuroimmunol Neuroinflamm 2019;6. pii: e602. 


\section{Figure 1 Caption}

A. Summary of the jCIDP age-specific prevalence rates ( $x$-axis) obtained in previous studies according to different geographical areas and age-groups (left y-axis) and diagnostic criteria (right y-axis, number in superscript refers to the original paper). B. jCIDP age-specific prevalence rates (x-axis) of the present study according to different age-groups (left y-axis) and diagnostic criteria (right y-axis). Legend: AAN= American Academy of Neurology; ENMC= European Neuromuscular Center criteria; EFNS/PNS=2006 and 2010 European Federation of Neurological Societies/Peripheral Nerve Society (EFNS/PNS) criteria. 
TABLE 1. Clinical and electrodiagnostic features of jCIDP cases. Five patients (nn. 1, 3, 4, 5 and 7) fulfil all diagnostic criteria

\begin{tabular}{|c|c|c|c|c|c|c|c|c|c|c|c|c|c|c|}
\hline n. & $\begin{array}{c}\text { Age at } \\
\text { onset } \\
\text { (sex) }\end{array}$ & $\begin{array}{c}\text { ENG } \\
\text { EFNS/ } \\
\text { PNS }\end{array}$ & $\begin{array}{l}\text { ENG } \\
\text { AAN }\end{array}$ & $\begin{array}{c}\text { ENG } \\
\text { ENMC }\end{array}$ & $\begin{array}{l}\text { Sup- } \\
\text { portive } \\
(\mathrm{CSF})\end{array}$ & $\begin{array}{l}\text { Sup- } \\
\text { portive } \\
\text { (MRI) }\end{array}$ & $\begin{array}{l}\text { Sup- } \\
\text { portive } \\
\text { (treat- } \\
\text { ment) }\end{array}$ & $\begin{array}{l}\text { EFNS/ } \\
\text { PNS } \\
\text { criteria }\end{array}$ & $\begin{array}{c}\text { AAN } \\
\text { criteria }\end{array}$ & $\begin{array}{l}\text { ENMC } \\
\text { criteria }\end{array}$ & $\begin{array}{l}\text { CIDP } \\
\text { subtype }\end{array}$ & Course & $\begin{array}{l}\text { Chronic } \\
\text { treatment }\end{array}$ & $\begin{array}{c}\text { ONLS } \\
\text { (follow- } \\
\text { up) }\end{array}$ \\
\hline 1 & $12 \mathrm{y}(\mathrm{m})$ & + & + & + & + & + & $-/+$ & definite & + & $\begin{array}{l}\text { confir- } \\
\text { med }\end{array}$ & $\begin{array}{l}\text { asymme- } \\
\text { tric } \\
\text { (MADSA } \\
\text { M) }\end{array}$ & progr & $\begin{array}{l}\text { steroids } \\
+ \text { IVIG }\end{array}$ & $\begin{array}{c}6 \\
(9 y)\end{array}$ \\
\hline 2 & $15 \mathrm{y}(\mathrm{m})$ & + & - & & + & n.d. & + & $\begin{array}{l}\text { proba- } \\
\text { ble }\end{array}$ & - & & typical & mono & - & $\begin{array}{c}2 \\
(3 y)\end{array}$ \\
\hline 3 & $17 y(m)$ & + & + & + & + & + & + & definite & + & $\begin{array}{l}\text { con- } \\
\text { firmed }\end{array}$ & typical & progr & steroids & $\begin{array}{c}4 \\
(3 y)\end{array}$ \\
\hline 4 & $9 \mathrm{y}(\mathrm{m})$ & + & + & + & + & + & + & definite & + & $\begin{array}{l}\text { con- } \\
\text { firmed }\end{array}$ & typical & relaps & - & $\begin{array}{c}4 \\
(10 y)\end{array}$ \\
\hline 5 & $5 y(f)$ & + & + & + & + & & + & definite & + & $\begin{array}{l}\text { con- } \\
\text { firmed }\end{array}$ & typical & progr & - & $\begin{array}{c}6 \\
(13 y)\end{array}$ \\
\hline 6 & $4 y(m)$ & + & & + & + & & + & definite & - & $\begin{array}{l}\text { con- } \\
\text { firmed }\end{array}$ & typical & relaps & $\begin{array}{c}\text { steroids }+ \\
\text { IVIG }\end{array}$ & $\begin{array}{c}5 \\
(15 y)\end{array}$ \\
\hline 7 & $16 \mathrm{y}(\mathrm{f})$ & + & + & + & + & + & + & definite & + & $\begin{array}{l}\text { con- } \\
\text { firmed }\end{array}$ & typical & relaps & IVIG & $\begin{array}{c}1 \\
(7 y)\end{array}$ \\
\hline 8 & $17 y(f)$ & - & - & & + & + & $\begin{array}{c}+ \\
\text { SSEPs }\end{array}$ & - & - & & $\begin{array}{l}\text { pure sen- } \\
\text { sory } \\
\text { (CISP) }\end{array}$ & mono & - & $\begin{array}{c}3 \\
(1 \mathrm{y})\end{array}$ \\
\hline
\end{tabular}

Abbreviations: $\mathrm{m}=$ male; $\mathrm{f}=$ female; $\mathrm{ENG}=$ electroneurographic diagnostic criteria; $\mathrm{EFNS} / \mathrm{PNS}=\mathrm{Eu}-$ ropean Federation of Neurological Societies/Peripheral Nerve Society 2006 and 2010-revised diagnostic criteria; ENMC= European Neuromuscular Center (ENMC) consortium criteria for juvenile CIDP; AAN= American Academy of Neurology, 1991, diagnostic criteria. $\mathrm{CSF}=$ cerebrospinal fluid; MRI= magnetic resonance imaging; MADSAM= multifocal acquired demyelinating sensory and motor neuropathy $;{ }^{1} \mathrm{CISP}=$ chronic immune sensory polyradiculopathy $;{ }^{1}$ progr= progressive; mono= monophasic; relaps= relapsing; SSEPs= somatosensory evoked potentials. IVIG= intravenous immunoglobulins; ONLS= Overall Neuropathy Limitation Scale. ${ }^{11}$ 